\title{
Deformed Skyrme Crystals
}

\author{
J. Silva Lobo* \\ Department of Mathematical Sciences, \\ Durham University, \\ Durham DH1 3LE
}

\begin{abstract}
The Skyrme crystal, a solution of the Skyrme model, is the lowest energy-percharge configuration of skyrmions seen so far. Our numerical investigations show that, as the period in various space directions is changed, one obtains various other configurations, such as a double square wall, and parallel vortex-like solutions. We also show that there is a sudden "phase transition" between a Skyrme crystal and the charge 4 skyrmion with cubic symmetry as the period is gradually increased in all three space directions.
\end{abstract}

PACS 12.39.Dc, 11.10.Lm, 11.27.+d

*email address: j.i.silva-lobo@durham.ac.uk 


\section{Introduction}

The Skyrme model was originally proposed by Tony Skyrme in 1961 [1]. It is a theory of nuclear matter in which the fundamental building blocks are pion fields. It was later shown [2, 3] that it can be regarded as a low-energy approximation to QCD, which becomes more accurate in the large $N_{c}$-limit, where $N_{c}$ is the number of quark colours. The (topologically non-trivial) solutions of this theory are called skyrmions. There is a special way of arranging them in order to obtain the smallest known value of the energy per baryon number seen so far [4], known as the Skyrme crystal. This is an infinite, triply-periodic, arrangement of half-skyrmions [5, 6, 7, 8, 9, 10]. In order to study Skyrme crystals, we impose periodic boundary conditions, with period $L$, on the Skyrme field in all three directions. The skyrmions are therefore defined on a 3 -torus $T^{3}$.

Skyrme crystals were originally proposed by Klebanov [5] as a model for dense nuclear matter, such as that found in neutron stars. At the time, the behaviour of two wellseparated skyrmions was already known [1] - an important feature being that these are maximally-attracted when one is rotated with respect to the other by $180^{\circ}$ about a line perpendicular to the line connecting them. Klebanov believed that an interesting extension of this idea would be to have an array of skyrmions, where any skyrmion would be attracted by its nearest neighbours. A graceful way of achieving this, Klebanov showed, would be to arrange the skyrmions in a simple-cubic lattice with appropriate rotations applied to all the nearest neighbours of a chosen skyrmion. Moreover, if the skyrme fields are to have the correct periodicity in all three directions, they must have symmetry elements that combine both spatial transformations as well as isospin transformations acting on the individual fields (more on this later in the introduction).

Klebanov also showed that there is a minimum in the energy per baryon number of the lattice for a certain value of the period $L$. In other words, there is a preferred size of the fundamental cell. Later, Goldhaber and Manton showed [6] that there is a phase transition from a low-density simple-cubic lattice of skyrmions to a high-density body-centred lattice of half-skyrmions. However, it has since been shown by Kugler and Shtrikman [9, 10] and by Castillejo et al. [8] that the lowest energy per baryon configuration is that of a (highdensity) half-skyrmion phase corresponding to an initial (low-density) face-centred cubic (fcc) array of skyrmions.

An fcc array is one in which skyrmions with standard orientation are placed on the vertices of a cube and more skyrmions are placed on the face centres, but this time rotated by $180^{\circ}$ about an axis perpendicular to the face. Such a configuration produces 12 nearest neighbours (to a particular skyrmion), which are all in the attractive channel. If the origin 
is fixed at the centre of one of the unrotated skyrmions and the skyrme fields are given by $\Phi_{\beta}(\mathbf{x})=\left(\Phi_{1}(\mathbf{x}), \Phi_{2}(\mathbf{x}), \Phi_{3}(\mathbf{x}), \Phi_{4}(\mathbf{x})\right)$, then the fcc configuration would have spatial and isospin symmetries, as was alluded to above. The generators for these symmetries are listed in Table 1 [4]:

Table 1: Symmetry generators of an fcc array of skyrmions

\begin{tabular}{ccc}
\hline \hline Transformation Name & Spatial Transformation & Isospin Transformation \\
\hline $\mathcal{R}_{1}$ & $\left(x_{1}, x_{2}, x_{3}\right) \rightarrow\left(-x_{1}, x_{2}, x_{3}\right)$ & $\left(\Phi_{1}, \Phi_{2}, \Phi_{3}, \Phi_{4}\right) \rightarrow\left(-\Phi_{1}, \Phi_{2}, \Phi_{3}, \Phi_{4}\right)$ \\
$\mathcal{R}_{1,-}^{3}$ & $\left(x_{1}, x_{2}, x_{3}\right) \rightarrow\left(x_{2}, x_{3}, x_{1}\right)$ & $\left(\Phi_{1}, \Phi_{2}, \Phi_{3}, \Phi_{4}\right) \rightarrow\left(\Phi_{2}, \Phi_{3}, \Phi_{1}, \Phi_{4}\right)$ \\
$\mathcal{R}_{1,-}^{4}$ & $\left(x_{1}, x_{2}, x_{3}\right) \rightarrow\left(x_{1}, x_{3},-x_{2}\right)$ & $\left(\Phi_{1}, \Phi_{2}, \Phi_{3}, \Phi_{4}\right) \rightarrow\left(\Phi_{1}, \Phi_{3},-\Phi_{2}, \Phi_{4}\right)$ \\
$\mathcal{T}_{1+, 2+}$ & $\left(x_{1}, x_{2}, x_{3}\right) \rightarrow\left(x_{1}+L / 2, x_{2}+L / 2, x_{3}\right)$ & $\left(\Phi_{1}, \Phi_{2}, \Phi_{3}, \Phi_{4}\right) \rightarrow\left(-\Phi_{1},-\Phi_{2}, \Phi_{3}, \Phi_{4}\right)$ \\
\hline
\end{tabular}

The transformations listed here are $\mathcal{R}_{1}$ : a reflection in the $x_{1}$-axis, $\mathcal{R}_{1,-}^{3}$ : a "negative" three-fold rotation about the diagonal that goes from the origin to the opposite corner of the cube (defined as "1"), $\mathcal{R}_{1,-}^{4}$ : a "negative" four-fold rotation about the $x_{1}$-axis, and $\mathcal{T}_{1+, 2+}$ : a positive $L / 2$-translation in both the $x_{1}$-axis and the $x_{2}$-axis. Note that these are a subset of the possible transformations that can be carried out on an fcc lattice. For example, one can also have a "positive" three-fold rotation along the same diagonal, $\mathcal{R}_{1,+}^{3}$, given by the transformation: $\left(x_{1}, x_{2}, x_{3}\right) \rightarrow\left(x_{3}, x_{1}, x_{2}\right), \quad\left(\Phi_{1}, \Phi_{2}, \Phi_{3}, \Phi_{4}\right) \rightarrow\left(\Phi_{3}, \Phi_{1}, \Phi_{2}, \Phi_{4}\right)$. Another possible transformation could also be a "positive" four-fold rotation about the $x_{3}$-axis, $\mathcal{R}_{3,+}^{4}:\left(x_{1}, x_{2}, x_{3}\right) \rightarrow\left(-x_{2}, x_{1}, x_{3}\right), \quad\left(\Phi_{1}, \Phi_{2}, \Phi_{3}, \Phi_{4}\right) \rightarrow\left(-\Phi_{2}, \Phi_{1}, \Phi_{3}, \Phi_{4}\right)$.

There is an additional symmetry unique to the high-density phase of half-skyrmions. Its generator is given by:

Table 2: Additional symmetry generator of the high-density half-skyrmion phase

\begin{tabular}{ccc}
\hline \hline Transformation Name & Spatial Transformation & Isospin Transformation \\
\hline $\mathcal{T}_{1+}$ & $\left(x_{1}, x_{2}, x_{3}\right) \rightarrow\left(x_{1}+L / 2, x_{2}, x_{3}\right)$ & $\left(\Phi_{1}, \Phi_{2}, \Phi_{3}, \Phi_{4}\right) \rightarrow\left(-\Phi_{1}, \Phi_{2}, \Phi_{3},-\Phi_{4}\right)$
\end{tabular}

Note that this transformation involves a chiral $\mathrm{SO}(4)$, rather than just isospin $\mathrm{SO}(3)$ rotations displayed in Table 1 above and it can replace the $\mathcal{T}_{1+, 2+}$ transformation since that can be achieved through successive applications of $\mathcal{T}_{1+}$ and $\mathcal{T}_{2+}$.

In this letter, we will see that the field behaves in an interesting way as one changes the period of the crystal in different ways, for different directions. For example, if we start with the Skyrme crystal and then increase the period along all three space dimensions in the same way, one gets the familiar picture of the cubically-symmetric charge $Q=4$ skyrmion. We also show that the energy density of the $Q=4$ skyrmion displays certain 
similarities previously seen in the context of Skyrme chains, provided we have large $L_{x, y}$ (where $L_{x}=L_{y}$ are the periods in the $x_{1}-$ and $x_{2}$-directions) and small $L_{z}$ values. Skyrme chains are solutions of the Skyrme model, which are periodic in one space dimension. It has been shown 11 that soliton chains generally have constituents in the form of vortexantivortex pairs. These emerge when the period is small compared to the natural soliton size - a feature that we verify when the period in the z-direction is small compared to the other two space dimensions. When the period increases, the constituents tend to clump together, a feature that is also verified here.

A double Skyrme sheet [12], is also seen to emerge at small $L_{x, y}$ and large (and effectively infinite) $L_{z}$ values. It takes the form of a square lattice, an object analogous to the hexagonal "Skyrme domain wall" solution [13]. However, our system is periodic in all three directions, which means that the vacuum value on both sides of the Skyrme sheets $( \pm \infty$ in the $\mathrm{z}$-direction) is unique.

Finally, we describe what happens as one increases the period simultaneously in all three directions, starting with the Skyrme crystal, and show that there is a rapid transition between the Skyrme crystal and the $Q=4$ skyrmion with cubic symmetry. We show evidence which suggests that this is a second-order phase transition with an order parameter given by the period $L_{x, y, z}$ of the configuration (where $L_{x}=L_{y}=L_{z}$ ).

\section{The Skyrme Crystal}

\subsection{Background}

The static energy density of the Skyrme model is given by:

$$
\mathcal{E}=-\frac{1}{2} \operatorname{Tr}\left(L_{i} L_{i}\right)-\frac{1}{16} \operatorname{Tr}\left(\left[L_{i}, L_{j}\right]\left[L_{i}, L_{j}\right]\right),
$$

where $L_{i}=U^{-1} \partial U / \partial x^{i}, x^{i}=(x, y, z)$ are the spatial coordinates, and the field $U\left(x^{i}\right)$ is an $\mathrm{SU}(2)$-valued scalar field. The pions are grouped into this scalar field as follows:

$$
U=\Phi_{4}+i \Phi_{i} \sigma_{i}
$$

where $\Phi_{i}$ is a triplet of pion fields, $\sigma_{i}$ is the triplet of Pauli matrices, and $\Phi_{4}$ is an additional scalar field determined through the constraint: $U^{\dagger} U=\Phi_{\beta} \Phi_{\beta}=1$.

The energy $E$ is defined as

$$
E=\frac{1}{12 \pi^{2}} \int \mathcal{E} d x d y d z
$$


The scalar field $U$ is a map from $\mathbb{R}^{3}$, compactified at infinity, to $S^{3}$, the group manifold of $\mathrm{SU}(2)$. Skyrme identified the degree of this map, a topological invariant, with the baryon number, which is given by

$$
Q=\int \mathcal{Q} d x d y d z
$$

where

$$
\mathcal{Q}=\frac{1}{24 \pi^{2}} \varepsilon_{i j k} \operatorname{Tr}\left(L_{i} L_{j} L_{k}\right)
$$

is the topological charge density. The energy (3) satisfies the Faddeev-Bogomolny lower bound [14] $E \geq Q$.

An analytic approximation for the fields of the Skyrme crystal was proposed in [8]. It takes into account the $\mathrm{SO}(4)$ chiral symmetry as well as the $\mathrm{SO}(3)$ isospin symmetries. The fields are expressed as follows:

$$
\begin{aligned}
& \Phi_{4}=c_{1} c_{2} c_{3} \\
& \Phi_{1}=-s_{1}\left(1-\frac{s_{2}^{2}}{2}-\frac{s_{3}^{2}}{2}+\frac{s_{2}^{2} s_{3}^{2}}{3}\right)^{\frac{1}{2}} \text { and cyclic permutations, }
\end{aligned}
$$

where $s_{i}=\sin \left(2 \pi x^{i} / L\right)$ and $c_{i}=\cos \left(2 \pi x^{i} / L\right)$. It is a good approximation to the actual minimal-energy solution.

\subsection{Changing the periods $L_{x}=L_{y}$ and $L_{z}$}

In what follows, we take periodic boundary conditions in all three space directions - the periods will be specified in the relevant sections. The lattice spacings in the $x, y$, and $z$ directions are given by $h_{x}, h_{y}, h_{z}$ and the number of lattice points are given by $n_{x}, n_{y}, n_{z}$, yielding side-lengths $L_{x, y, z}=h_{x, y, z} * n_{x, y, z}$. We use a first-order finite-difference scheme and implement a full 3-dimensional numerical minimization of the energy using the conjugate gradient method ( $c f .[15]$ ).

Note that there is a numerical error associated with the finite lattice spacing. The way we approximate the errors in the energy, for a given configuration, is by comparing its topological charge (using numerical methods) with the "true" value of its charge. We assume the same errors in energy and charge since similar finite-difference methods are employed in calculating each of these values. We have noticed that higher differences in the lattice spacing in each space direction yield higher errors.

The initial condition that we start with for minimization is the approximate skyrme crystal of eight half-skyrmions, namely (6) and (7). After being minimized, the period in each direction for this initial configuration is then changed in a certain way (described in the relevant section) and then re-minimized. 


\subsubsection{From a 4-skyrmion to a square 2-wall}

The first case is the one for which the period in all three directions is large for the initial configuration and then the $L_{x, y}$ periods are reduced gradually. The initial period is given by $L_{x, y, z}=7.05$ (see Fig. 1) and then reduce $L_{x}=L_{y}$ by 1 each time.

We start by increasing the periods $L_{x}=L_{y}=L_{z}$ for the minimized Skyrme crystal from $L_{x, y, z}=4.7$ to 7.05 by increasing the value of $n_{x, y, z}$, for a certain value of $h_{x, y, z}$, and thus producing Fig.1(a). Afterwards, $n_{x, y, z}$ is kept constant and $L_{x, y, z}$ is reduced by 1 each time.

One can see that the translation symmetries, $\mathcal{T}_{i+/-}$, (where $i=\{1,2,3\}$ and in either direction $+/-$ ) of the high-density Skyrme crystal are broken in Fig.1(a), which is the $Q=4$ skyrmion, whereas all reflection symmetries, $\mathcal{R}_{i}$, three-fold rotations $\mathcal{R}_{1 / 2,+/-}^{3}$, and four-fold rotations $\mathcal{R}_{i,+/}^{4}$ remain unbroken - characteristic of cubic symmetry. In Fig. 1(b)-(f), $\mathcal{T}_{1+/-}$ and $\mathcal{T}_{2+/-}$ symmetries are regained (possibly due to the fact that we are "squeezing" the configuration in these directions), whereas $\mathcal{T}_{3+/}$ remains broken only for Fig. 1(b) and Fig. 1(c), due to the fact that these figures are not extended throughout the whole period in the $z$-direction. The reflection symmetries $\mathcal{R}_{i}$ are unbroken and the three-fold rotations $\mathcal{R}_{1 / 2,+/-}^{3}$ are broken in Fig. 1(b)-(f). The four-fold rotations $\mathcal{R}_{3,+/-}^{4}$ remain unbroken throughout, whereas $\mathcal{R}_{1 / 2,+/-}^{4}$ are broken in Fig. 1(b)-(f).

Note that a double Skyrme sheet configuration [12] emerges in Fig. 1(d). The separation between the sheets, which is calculated by measuring the distance between the energy density peaks as a function of $z$, remains constant as $L_{x, y}$ is reduced further and $L_{z}$ is kept constant at $L_{z}=7.05$. However, the energy decreases from a value of $E=4.36 \pm 0.09$ at $L_{x, y, z}=7.05$ down to a minimum of $E=4.20 \pm 0.05$ at $L_{x, y}=4.05$ and, finally, increases to $E=5.25 \pm 0.10$ at $L_{x, y}=2.05$.

The preferred configuration for this Skyrme sheet is to have $L_{x}=L_{y}$. For instance, if we fix $L_{y}=4.05$ and vary $L_{x}$ away from this value in either direction, we notice that the energy increases.

\subsubsection{From square 2-walls to vortices}

We now start with a large $L_{z}$ period, which will be reduced, and keep $L_{x, y}=4$ constant. The starting point is $L_{z}=7$, which is then reduced by 1 each time. In this section, $n_{x}=n_{y}=n_{z}$ are kept constant, producing different $h_{z}$ values each time $L_{z}$ is changed.

The initial condition (eqs. (6) and (7)) is first minimized for the periods $L_{x, y}=4$ and $L_{z}=7$, producing the double Skyrme sheet configuration seen in Fig. 2(a). The only broken symmetries associated with this configuration, which is seen to persist in Fig. 2(b) 

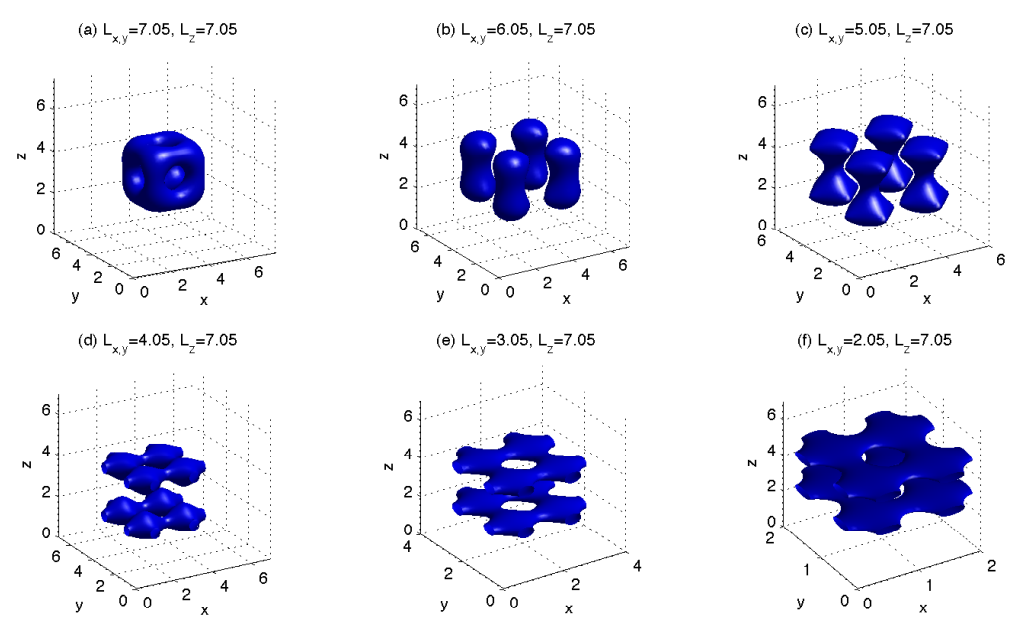

Figure 1: Energy density isosurfaces with surface value given by $0.5 * \mathcal{E}_{\max }$, where $\mathcal{E}_{\max }$ is the maximum value of the energy density (all isosurfaces in subsequent figures have the same surface value). The first isosurface corresponds to the $Q=4$ skyrmion for the periods $L_{x, y, z}=7.05$. Each successive picture has $L_{x, y}$ reduced by 1 and $L_{z}$ is kept constant at 7.05 .

and Fig. 2(c), are $\mathcal{R}_{1 / 2,+/-}^{4}$ and $\mathcal{R}_{1 / 2,+ \text { - }^{3}}^{3}$ These are regained in Fig. 2(d), which is a (non-minimal) Skyrme crystal configuration. As the $L_{z}$ period is decreased further, we notice the appearance of vortex-like structures, which are periodic in the $z$-direction (Fig. 2(e) and Fig. 2(f)). The symmetries associated with these configurations are the same as those in Figs. 2(a)-(c) and, in fact, the translation symmetry in the $z$-direction, $\mathcal{T}_{3+/-}$, becomes continuous for Fig. 2(f), since there is no longer any noticeable $z$-dependence. This ties into the subject of Skyrme chains, which has been explored in [11].

The energy of the isosurfaces decreases from a value of $E=4.22 \pm 0.05$ at $L_{z}=7$, down to a minimum of $E=4.17 \pm 0.04$ at $L_{z}=5$ (where the isosurfaces are still in the form of a double square wall), then back up to $E=4.89 \pm 0.05$ at $L_{z}=2$.

\subsubsection{From square 2-walls to vortices, via crystal}

In this section, we show how one can transform a double Skyrme sheet configuration into the 4 -vortex configuration discussed in the previous section, by changing both $L_{x}=L_{y}$ and $L_{z}$ (rather than just $L_{z}$ ), and going through an intermediate, minimal, Skyrme crystal state, as can be seen in Fig. 3. The initial period is $L_{x, y}=2.7$ and $L_{z}=10.7$. The former is then increased by 1 and the latter decreased by 3, two consecutive times, producing Figs. $3(\mathrm{~b})-(\mathrm{c})$. The changes in the periods are then swapped, increasing $L_{x, y}$ by 3 and decreasing 

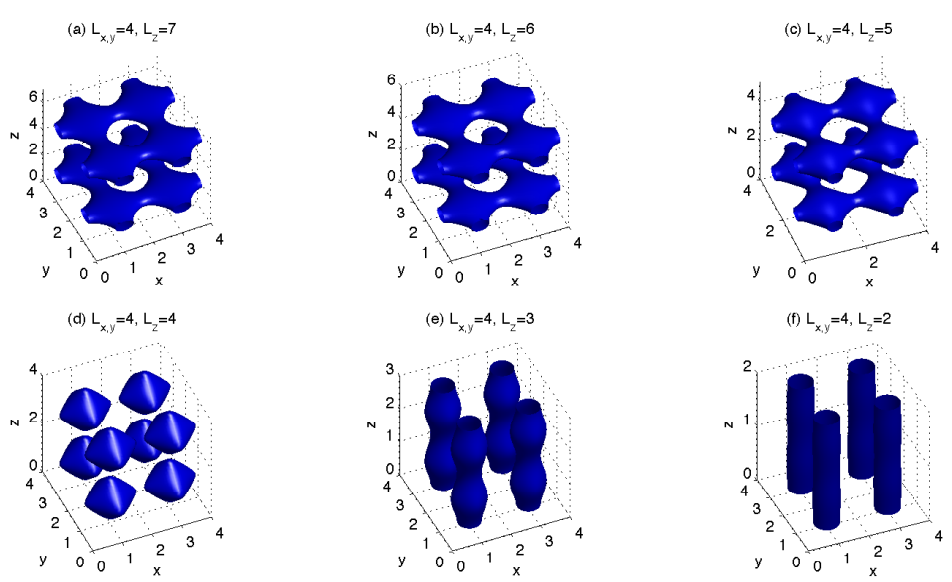

Figure 2: Energy density isosurfaces corresponding to the $Q=4$ double Skyrme sheet configuration, where $L_{x, y}=4$ remains fixed and where $L_{z}=7$ is reduced by 1 in each successive picture, transforming the double skyrme sheet into a (non-minimal) Skyrme crystal and thereafter into a parallel 4-vortex structure.

$L_{z}$ by 1 , for two consecutive times, producing Figs. 3(d)-(e). Here, $n_{x}=n_{y}=n_{z}$ are kept constant, producing different $h_{x}=h_{y}$ and $h_{z}$ values, each time the periods are changed.

We start by minimizing the approximate Skyrme crystal (eqs. (6) and (7)) for the periods $L_{x, y}=2.7$ and $L_{z}=10.7$, producing the double Skyrme sheets discussed in the previous sections (with the same symmetries) in Fig. 3(a). As the periods are changed, as described above, the Skyrme sheets still persist in Fig. 3(b), and change into the (minimalenergy) Skyrme crystal in Fig. 3(c) (with all its associated symmetries as described in the introduction). As $L_{x, y}$ increase and $L_{z}$ decreases further, the Skyrme crystal changes into a 4-vortex structure, Figs. 3(d)-(e), which have less of a $z$-dependence than the ones in Figs. 2(e)-(f), respectively.

The Skyrme crystal has the minimum energy, with $E=4.13 \pm 0.04$, followed by the square 2 -walls at $L_{x, y}=3.7$ and $L_{z}=7.7$ with $E=4.26 \pm 0.06$. The final picture, the 4 -vortex configuration with the smallest $L_{z}$-value, has the highest energy, with $E=$ $4.77 \pm 0.17$, which follows from the fact that Skyrme chains "prefer" to be closer together in the $x, y$-directions [11].

Thus far, we have changed the periods in the three space directions in different ways by decreasing $L_{x, y}$ (or keeping it constant) and keeping $L_{z}$ constant (or decreasing it) or changing all three periods at different rates at the same time. We now turn to the case where we increase (or decrease) all three periods simultaneously at the same rate. 

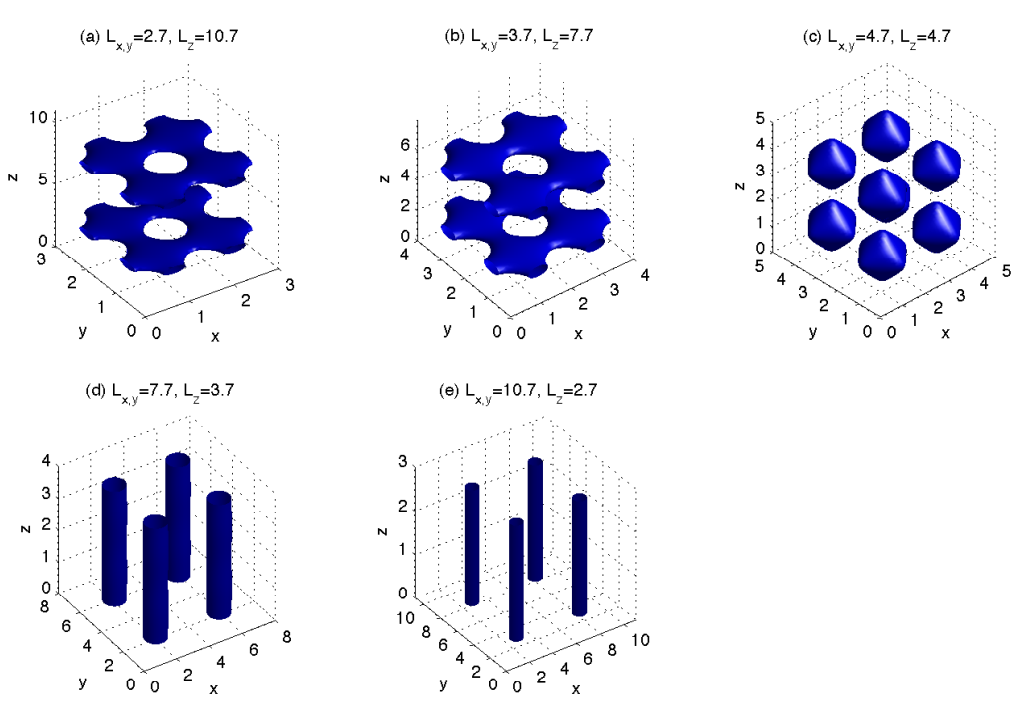

Figure 3: Energy density isosurfaces, which show a transition from a pair of Skyrme sheets to a 4 -vortex configuration. Subsequent pictures have $L_{x, y}$ and $L_{z}$ changing in such a way that their values are swapped halfway through the transition.

\subsubsection{From the Skyrme crystal to the $Q=4$ skyrmion}

It turns out that an interesting feature of the $Q=4$ system is uncovered when one starts from the minimal-energy Skyrme crystal configuration at $L_{x, y, z}=4.7$ and then increases the periods simultaneously until the individual half-skyrmions coalesce.

These half-skyrmions clump in a sudden fashion when one perturbs the system in a certain way. We perturbed it by removing one lattice site through the middle of the configuration in all three directions, thereby "squeezing" the half-skyrmions together in all directions. Therefore, in order to see them coalescing, one needs to increase the periods very gradually. This appears to happen in the range $L_{x, y, z}=6.07-6.09$ as can be seen in Fig. 4, where the first picture has periods $L_{x, y, z}=4.7$ and the following three correspond to the range just mentioned $]^{1}$ Compare this with Fig. 5, where the periods are increased in larger steps.

The sudden merging of the half-skyrmions can be visualized in a different fashion by taking the maximum value of the difference in the energy densities of the fields $\mathcal{E}\left[\Phi_{\beta}\left(x^{j}\right)\right]$ under a certain symmetry transformation, in this case a translation of $L_{x} / 2$ in the $x$-direction, which is half the size of the fundamental cell, and dividing by the maximum value of the energy density, i.e. $\Delta_{1}=\left(\mathcal{E}\left[\Phi_{\beta}\left(x^{j}\right)\right]-\mathcal{E}\left[\Phi_{\beta}\left(x^{j^{\prime}}\right)\right]\right)_{\max } / \mathcal{E}_{\max }$, where $x^{j^{\prime}}=x^{j}-L_{x} / 2$. We

\footnotetext{
${ }^{1}$ It is worth mentioning that the value of this range changes when the bin size is changed - we used the value $n_{x, y, z}=32$. However, the general feature that the half-skyrmions coalesce rapidly remains the same.
} 

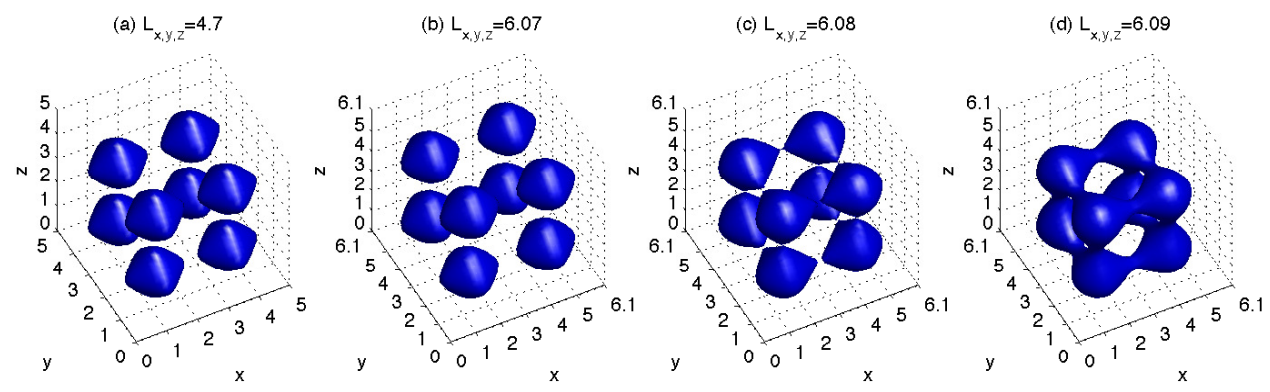

Figure 4: The energy density isosurface of the $Q=4$ Skyrme crystal at $L_{x, y, z}=4.7$. The periods are then increased gradually from $L_{x, y, z}=6.07$ to $L_{x, y, z}=6.09$.
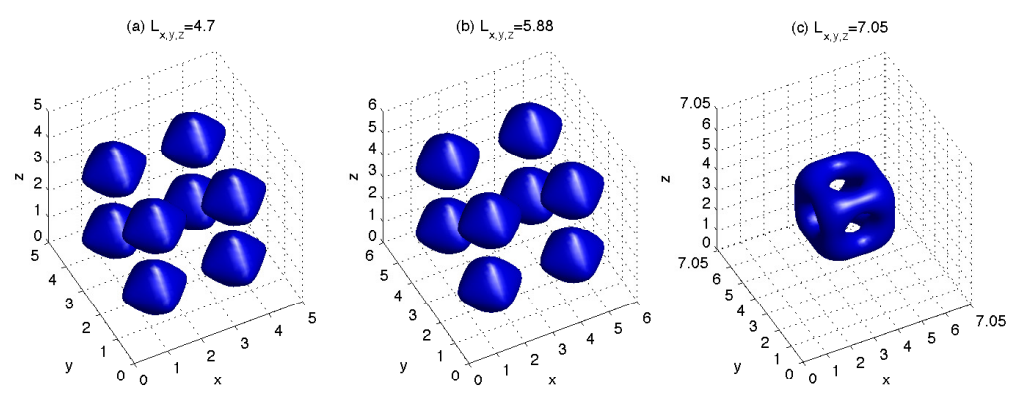

Figure 5: The first figure corresponds to the energy density isosurface of the Skyrme crystal for the periods $L_{x, y, z}=4.7$, the second figure has periods $L_{x, y, z}=5.88$, and the third one has $L_{x, y, z}=7.05$

then plot this as a function of the period $L_{x, y, z}$ - the reason we do this is that, as soon as the half-skyrmions start to coalesce, they will no longer be $L_{x} / 2$-periodic. For the Skyrme crystal, this difference is seen to be essentially zero and it then starts to increase as the half-skyrmions begin to coalesce as can be seen in Fig. 6

This jump in the asymmetries of the crystal is analogous to a phase transition in thermodynamics. There is a sudden transition from a crystalline phase, which has more symmetries, such as chiral SO(4) symmetries for the Skyrme crystal, to a phase with less symmetries, such as $\mathrm{SO}(3)$ isospin symmetries for the $Q=4$ cubic-shaped skyrmion. We expect the transition to become more pronounced as the number of bins increases - as can be seen in Fig. 6, when $n_{x, y, z}$ is increased from 32 to 36 , which starts to resemble a step function. It should be noted that the $\Delta_{1}$ values are independent of which $L_{x, y, z}$ value one starts with, which hints to a lack of hysteresis in the system. Extending the analogy with phase transitions, this lack of hysteresis strongly suggests a second-order phase transition (cf. [16]), with no latent heat, and with the period $L_{x, y, z}$ as the order parameter. 


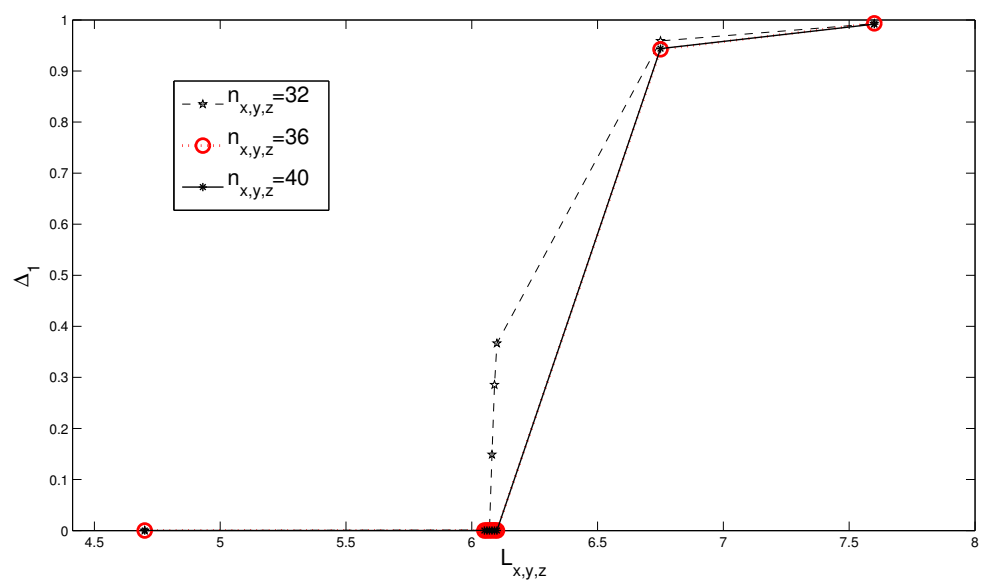

Figure 6: Difference in the value of the energy density of the Skyrme fields under an $L_{x} / 2$-translation in the $x$-direction divided by $\mathcal{E}_{\max }$, for different values of the period $L_{x, y, z}$. Note that as the bin size decreases (or as $n$ increases), the plot begins to look more like a step function.

\section{Concluding Remarks}

We have seen that changing the periods of the Skyrme crystal, away from the values which minimize its energy, has allowed us to find new configurations - such as the double square lattice of "Skyrme sheets" - as well as unearthing unforeseen connections with Skyrme chains. The Skyrme sheets are produced when the period $L_{x, y}$ is small compared with $L_{z}$. Our results [12] have shown that these are more energetically favourable than the double hexagonal case, which makes them a prime candidate for use as building blocks for largecharge configurations. If one then goes to the other limit of large $L_{x, y}$ and small $L_{z}$ one gets a series of four vortex-like charge $Q=1$ objects, which is related to the subject of Skyrme chains [11]. As the periods are changed and we go through these different configurations, we have seen that these generally merge and separate in the directions in which one is changing the period, for example, the merging of the half-skyrmions in the $z$ direction as the period is decreased along this direction.

We have also noticed that the phase transition from the Skyrme crystal to a charge $Q=4$ skyrmion is likely to be second-order, due to the lack of hysteresis in the system, and with an order parameter given by the period $L_{x, y, z}$.

Note that the deformations considered here have all been along one or more of the edges of the Skyrme crystal, keeping it rectangular. An interesting extension, which we plan to investigate, would be to see if any new structures emerge when one applies more general affine transformations, such as a stretch along a diagonal of the crystal. 
Acknowledgment. I would like to thank Professor Richard Ward for helping me with the editing process, Professor Nick Manton for the useful and interesting discussions about hysteresis, and Carlos Silva Platt for his support.

\section{References}

[1] T. H. R. Skyrme, "A Nonlinear field theory," Proc. Roy. Soc. Lond. A 260 (1961) 127.

[2] E. Witten, "Current Algebra, Baryons, And Quark Confinement," Nucl. Phys. B 223 (1983) 433.

[3] E. Witten, "Baryons In The 1/N Expansion," Nucl. Phys. B 160 (1979) 57.

[4] N. Manton and P. Sutcliffe. Topological Solitons. Cambridge University Press, 2004, ISBN 0-521-83836-3.

[5] I. R. Klebanov, "Nuclear Matter In The Skyrme Model," Nucl. Phys. B 262, 133 (1985).

[6] A. S. Goldhaber and N. S. Manton, "Maximal Symmetry Of The Skyrme Crystal," Phys. Lett. B 198, 231 (1987)

[7] A. D. Jackson and J. J. M. Verbaarschot, "Phase Structure Of The Skyrme Model," Nucl. Phys. A 484, 419 (1988)

[8] L. Castillejo, P. S. J. Jones, A. D. Jackson, J. J. M. Verbaarschot and A. Jackson, "Dense Skyrmion Systems," Nucl. Phys. A 501, 801 (1989)

[9] M. Kugler and S. Shtrikman, "A New Skyrmion Crystal," Phys. Lett. B 208, 491 (1988)

[10] M. Kugler and S. Shtrikman, "Skyrmion Crystals and Their Symmetries," Phys. Rev. D 40, $3421(1989)$

[11] D. Harland and R. S. Ward, "Chains of Skyrmions," JHEP 0812, 093 (2008) arXiv:0807.3870 [hep-th]].

[12] J. Silva Lobo and R. S. Ward, "Skyrmion Multi-Walls," J. Phys. A 42 (2009) 482001 arXiv:0910.5457 [hep-th]]. 
[13] R. A. Battye and P. M. Sutcliffe, "A Skyrme lattice with hexagonal symmetry," Phys. Lett. B 416, 385 (1998) arXiv:hep-th/9709221.

[14] L. D. Faddeev, "Some Comments On The Many Dimensional Solitons," Lett. Math. Phys. 1, 289 (1976).

[15] W.H. Press, B.P. Flannery, S.A. Teukolsky, and W.T. Vetterling. Numerical Recipes in FORTRAN 77. Cambridge University Press, 1992, ISBN 052143064X.

[16] A. Visintin. Differential Models of Hysteresis. Springer-Verlag Berlin Heidelberg, 1994, ISBN 3-540-54793-2. 